THE ASTRONOMICAL JOURNAL, 118:1806-1813, 1999 October

\title{
CEPHEID COLOR-TEMPERATURE RELATIONS
}

\author{
ANNe M. Fry ${ }^{1,2}$ and Bruce W. CARneY ${ }^{1}$ \\ Department of Physics and Astronomy, University of North Carolina at Chapel Hill, Chapel Hill, NC 27599-3255; \\ anne@envy.astro.unc.edu,bruce@sloth.astro.unc.edu \\ Received 1997 June 5 ; accepted 1999 June 16
}

\begin{abstract}
Using our 1997 work in spectroscopic temperatures, as well as published Cepheid photometry, we derive color-temperature relations for a set of 13 Cepheids in Galactic clusters. The colors and temperatures used are individual values taken at different phases, rather than mean values averaged over the pulsational cycle. The observed colors are corrected for both reddening and metallicity. The effect of gravity on the colors is small. We find that $B-V$ and $V-R$ are the best colors to use to determine a Cepheid photometric temperature scale. These color-temperature relations will be useful for future Cepheid Baade-Wesselink studies and for abundance analyses of more Galactic and extragalactic Cepheids.

Keywords: Cepheids - stars: fundamental parameters
\end{abstract}

\section{INTRODUCTION}

In a recent paper (Fry \& Carney 1997), we presented abundance analyses of 101 spectra for 23 Galactic Cepheids to remove metallicity effects from main-sequence fitting used to calibrate the zero point of the period-luminosity (P-L) relation. Using our spectroscopically derived temperatures and published photometry, we are able to form color-temperature relations, which have a wide variety of uses, for the Cepheids in open clusters.

For example, Baade-Wesselink analyses offer us an opportunity to derive absolute and relative distances to Cepheids, but the method requires good color-temperature relations. One of our own goals, and the subject of future papers, is to test the metallicity sensitivity of the P-L relation by deriving distances to Cepheids in the Galaxy, the Large Magellanic Cloud $([\mathrm{Fe} / \mathrm{H}] \approx-0.4)$, and the Small Magellanic Cloud $([\mathrm{Fe} / \mathrm{H}] \approx-0.6)$. Furthermore, since one of the key debates is the distance scales derived via Cepheids versus those from RR Lyrae stars, we need to know how well the Baade-Wesselink method works when applied to each type of variable. Two RR Lyrae variables in the globular cluster M5 have been analyzed using the Baade-Wesselink method (Storm, Carney, \& Latham 1994), and the cluster also contains some long-period Cepheids. Since these Cepheids have similar temperatures and gravities (but not luminosities) to Population I Cepheids, this will provide a good test of the applicability of the BaadeWesselink method to both types of stars.

Another opportunity will be provided by using highresolution but low signal-to-noise $(\mathrm{S} / \mathrm{N})$ spectra to estimate mean metallicities of Cepheids throughout the Galaxy and, indeed, the Local Group, following the methods described by Carney et al. (1987) and Laird, Carney, \& Latham (1988). This requires estimating the stellar temperature, which may

${ }^{1}$ Visiting Astronomer, Kitt Peak National Observatory and Cerro Tololo Inter-American Observatory, National Optical Astronomy Observatories, operated by the Association of Universities for Research in Astronomy, Inc., under cooperative agreement with the National Science Foundation.

${ }^{2}$ Current address: Department of Astronomy, Case Western Reserve University, Cleveland, $\mathrm{OH} 44106$. be obtained from photometry, and some means of estimating the reddening (as discussed below). Indeed, a good color-temperature relation will prove valuable for any spectroscopic analyses for which the $\mathrm{S} / \mathrm{N}$ is too low or the wavelength coverage is inadequate for a reliable spectroscopic estimate of the temperature to be derived.

Many other authors have derived Cepheid colortemperature relations. Oke (1961a, 1961b) and Teays (1986) used spectrophotometry to determine the temperatures of the Cepheids in their studies. Flower (1977) and BöhmVitense (1981) used a variety of direct and indirect temperature determinations to derive a supergiant colortemperature scale. Our study is the first to use Cepheid excitation temperatures for a large, homogenous sample of Cepheids to derive a Cepheid color-temperature relation. The Cepheid excitation temperatures were determined using high-resolution, high-S/N spectra, requiring that the calculated abundance not be a function of excitation potential. To test our spectroscopic temperature scale, we observed most of the Cepheids at more than one pulsational phase and verified that the derived abundance did not change sfignificantly over the pulsational cycle (Fry \& Carney 1997).

\section{TEMPERATURE INDICATORS}

Which color index is a good temperature indicator for Cepheids? In principle, a color index among those with the highest sensitivity to temperature (e.g., $V-K$ ) is preferred. However, Baade-Wesselink analyses impose the additional constraint that the distance between the line-forming layers, which yield pulsational velocities, and the continuumforming layers, which yield colors and temperatures, apparent luminosities, and apparent angular diameters, must be small and constant throughout the pulsational cycle to prevent systematic errors. Similarly, the distances between the continuum-forming layers at the wavelengths of the filter bandpasses should be zero, preferably, or at least small and constant.

Using ATLAS9 (Kurucz 1993) to calculate surface fluxes, we estimate the depths of formation of a star's continuum and of the lines for each of the bandpasses $B, V, R, I$, and $K$. The depth of formation of the continuum is taken to be $\tau=1.0$. In Figure 1, we estimate the depth of formation in 


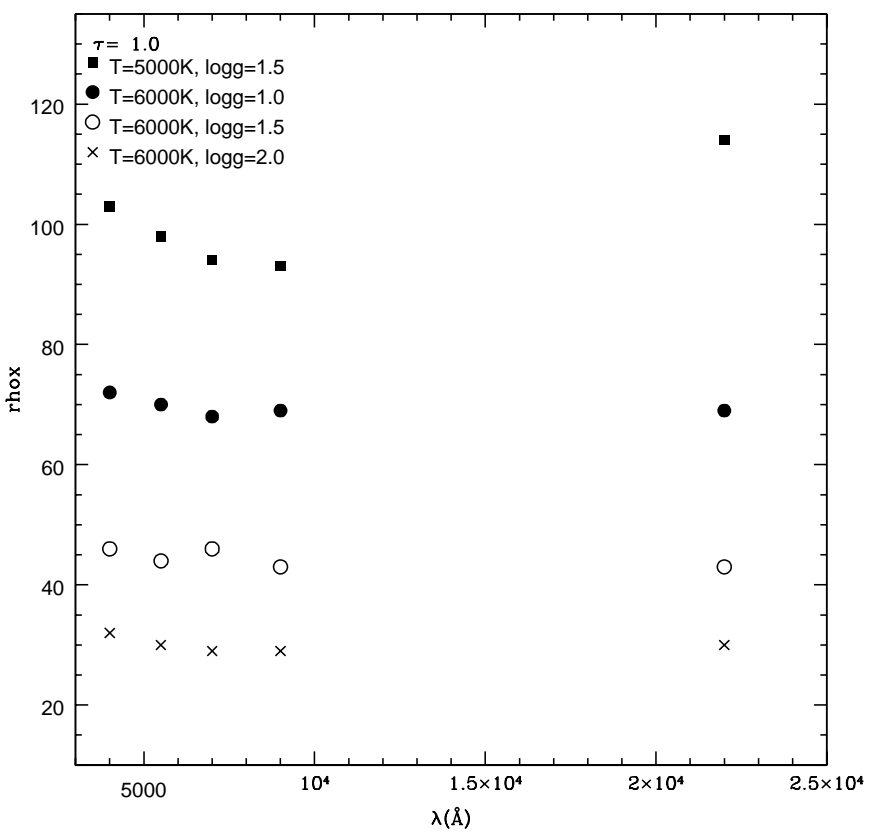

FIG. 1.-Plot of rhox vs. $\lambda$ for $\tau=1.0$ and $T_{\text {eff }}=5000 \mathrm{~K}, \log g=1.5$ and $T_{\text {eff }}=6000 \mathrm{~K}, \log g=1.0,1.5,2.0$.

terms of a mass variable

$$
\operatorname{rhox}=-\int \rho d x .
$$

Figure 1 shows the depths of formation as a function of the bandpass central wavelength $\lambda$ for several different models appropriate for Cepheids. Comparing the $\log g=1.5$, $T_{\text {eff }}=5000 \mathrm{~K}$, and $T_{\text {eff }}=6000 \mathrm{~K}$ points, we see which colors should make good temperature indicators. A good temperature indicator is one for which

$$
\operatorname{rhox}_{\lambda 1} / \operatorname{rhox}_{\lambda 2} \neq f\left(T_{\text {eff }}\right) \text {. }
$$

$B-V$ and $V-R$ appear to satisfy this criterion.

As a consequence of either differing depths of formation or the flux excess in the blue spectral regions during the expansion phases, $V-K$ has been the preferred temperature indicator for RR Lyrae variables (see Jones 1988 for a detailed discussion). For the Cepheids, however, $V-K$ does not seem to satisfy equation (2). At $\tau=$ 1.0 and $T_{\text {eff }}=5000 \mathrm{~K}$,

$$
\operatorname{rhox}_{V} / \operatorname{rhox}_{K} \approx 0.85 \text {. }
$$

However, at $T_{\text {eff }}=6000 \mathrm{~K}$,

$$
\operatorname{rhox}_{V} / \operatorname{rhox}_{K} \approx 1.02
$$

\section{FORMING COLOR-TEMPERATURE RELATIONS}

We wish to derive a relation between effective temperature and the $B-V$ and $V-R$ color indexes. Because both have some sensitivity to metallicity, we derive the relations for a fixed metallicity, in this case solar. We must correct the color indexes for reddening and then make the corrections to reflect a solar metallicity. For cluster Cepheids, the reddenings are straightforward, determined from $U B V$ photometry of early-type cluster members. In Table 1, we list the Cepheids, as well as their metallicities and reddenings.

For the field stars, the reddening corrections rely on a relation between dereddened colors and period, but this relation must be shifted to the metallicity of each field star so that metallicity differences among the Cepheids do not introduce systematic errors. We have chosen not to include the field Cepheids in our color-temperature calibration.

\subsection{Reddening}

To form color-temperature relations, we first need an accurate estimate of the reddening for each Cepheid. We adopt the reddenings derived from $U B V$ photometry of the upper main-sequence stars, corrected for the Cepheids' colors:

$$
E(B-V)_{\text {Cepheid }}=E(\mathrm{OB})\left[0.98-0.09\left\langle B_{0}\right\rangle-\left\langle V_{0}\right\rangle\right],
$$

where $E(\mathrm{OB})$ is the reddening determined from the upper main-sequence stars and $\left\langle B_{0}\right\rangle-\left\langle V_{0}\right\rangle$ is the intensityweighted mean color of the Cepheid (Feast \& Walker 1987). Reddenings derived using the upper main-sequence stars are insensitive to metallicity. The metallicities and reddenings of the Cepheids are listed in Table 1.

\subsection{Correcting the Colors to Solar Metallicity}

\begin{tabular}{|c|c|c|c|c|}
\hline \multirow[b]{2}{*}{ CEPHEID } & \multirow[b]{2}{*}[\mathrm{Fe}/\mathrm{H}]{} & \multicolumn{2}{|c|}{$E(B-V)$} & \multirow[b]{2}{*}{ REFERENCE } \\
\hline & & OB Stars & Adopted & \\
\hline CF Cas ....... & $-0.20 \pm 0.02$ & 0.540 & 0.500 & Fry 1997 \\
\hline DL Cas ....... & $+0.05 \pm 0.01$ & 0.470 & 0.433 & Turner et al. 1992 \\
\hline V Cen .......... & $-0.14 \pm 0.02$ & 0.310 & 0.287 & Claria et al. 1991 \\
\hline T Mon ......... & $+0.09 \pm 0.02$ & 0.179 & 0.159 & Feast \& Walker 1987 \\
\hline CV Mon...... & $-0.05 \pm 0.06$ & 0.719 & 0.667 & Feast \& Walker 1987 \\
\hline S Nor ......... & $-0.03 \pm 0.02$ & 0.190 & 0.173 & Fry 1997 \\
\hline TW Nor ...... & $+0.03 \pm 0.03$ & 1.234 & 1.123 & Feast \& Walker 1987 \\
\hline U Sgr ......... & $+0.01 \pm 0.03$ & 0.500 & 0.460 & Fry 1997 \\
\hline WZ Sgr ........ & $-0.15 \pm 0.03$ & 0.560 & 0.504 & Turner et al. 1993 \\
\hline EV Sct ........ & $-0.37 \pm 0.03$ & 0.557 & 0.516 & Feast \& Walker 1987 \\
\hline SZ Tau ....... & $-0.01 \pm 0.03$ & 0.290 & 0.272 & Turner 1992 \\
\hline SW Vel ....... & $-0.08 \pm 0.03$ & 0.350 & 0.318 & Turner et al. 1993 \\
\hline SV Vul........ & $+0.06 \pm 0.04$ & 0.438 & 0.389 & Feast \& Walker 1987 \\
\hline
\end{tabular}

We must make small corrections for all the variables' color indexes to reflect a common metallicity. To remove

TABLE 1

CepheId Reddenings and Metallicities 
the metallicity dependence from our Cepheid colortemperature relations, we used Kurucz's grid of synthetic colors (Kurucz 1993) to calculate, for a given temperature and gravity, the difference in color between a star with a given metallicity and a star with solar metallicity. The color corrections are listed as a function of temperature and gravity in Table 2 . We formed the color corrections by interpolating in $T_{\text {eff }}, \log g$, and $[\mathrm{Fe} / \mathrm{H}]$ and then applying the corrections to published Cepheid photometry (Moffett \& Barnes 1980, 1984; Welch et al. 1984; Laney \& Stobie 1992) at the phases of observation. The spectroscopic temperatures, metallicity-corrected colors, and metallicity corrections are listed in Table 3.

Least-squares fits were made between the dereddened, metallicity-corrected colors listed in Table 3 and the corresponding effective temperatures given in Table 4 of Fry \& Carney (1997). We show the fits in Figures 2 and 3, where

$$
\Theta_{\text {eff }}=5040 \mathrm{~K} / T_{\text {eff }} \text {. }
$$

For our data, there are errors both in our spectroscopic temperature and in the photometry (observational and reddening errors). To include errors in temperature and photometry, we did $y=y(x)$ fits and $x=x(y)$ fits. We inverted the $x=x(y)$ fits to form $y=y(x)$ and then formed a bisector fit from the two $y(x)$ fits (see Isobe et al. 1990). All three fits are shown in the figures. We find the following bisector fits:

$$
\begin{aligned}
& \Theta_{\text {eff }}=(0.716 \pm 0.015)+(0.225 \pm 0.017)(B-V)_{0}, \\
& \Theta_{\text {eff }}=(0.609 \pm 0.024)+(0.429 \pm 0.034)(V-R)_{0} .
\end{aligned}
$$

To see how well our color-temperature relations work, we can use equation (7) and Cepheid photometry to work backward. We calculate $\Delta T=T_{\text {spect }}-T_{B-V}$ for each spectrum: $\langle\Delta T\rangle=0 \pm 157 \mathrm{~K}$. (Since the formal temperature error in the fit is $\pm 125 \mathrm{~K}$ and in the spectroscopic temperature estimates is $\pm 100 \mathrm{~K}$ [cf. Fry \& Carney 1997], we

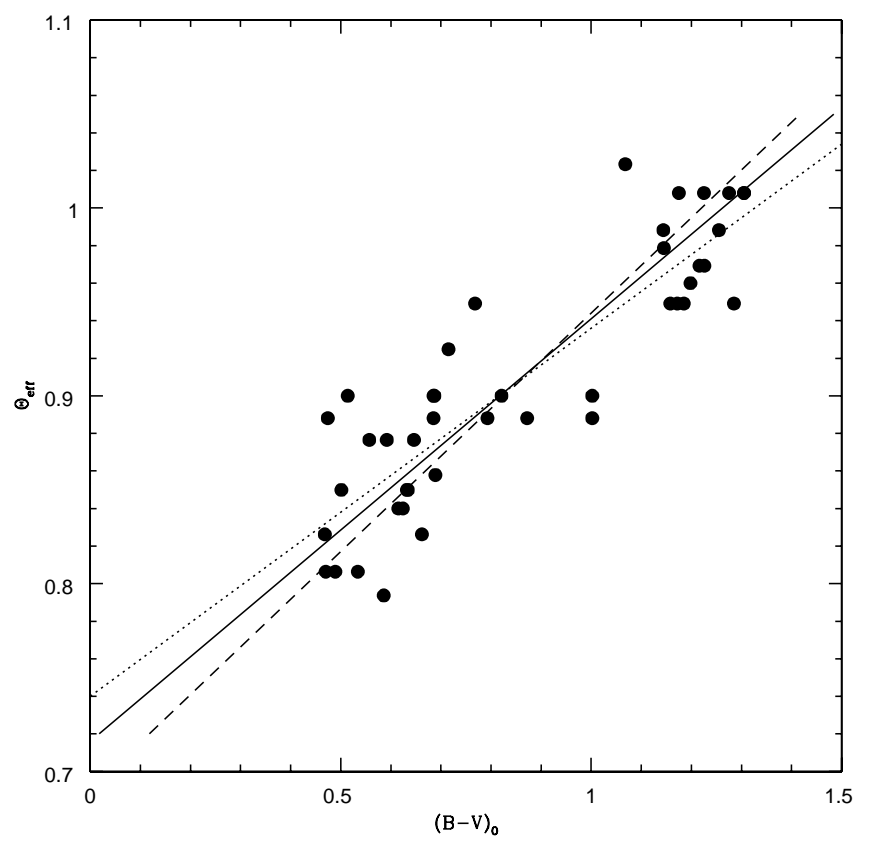

FIG. 2. $-\Theta_{\text {eff,spect }}$ vs. $(B-V)_{0}$

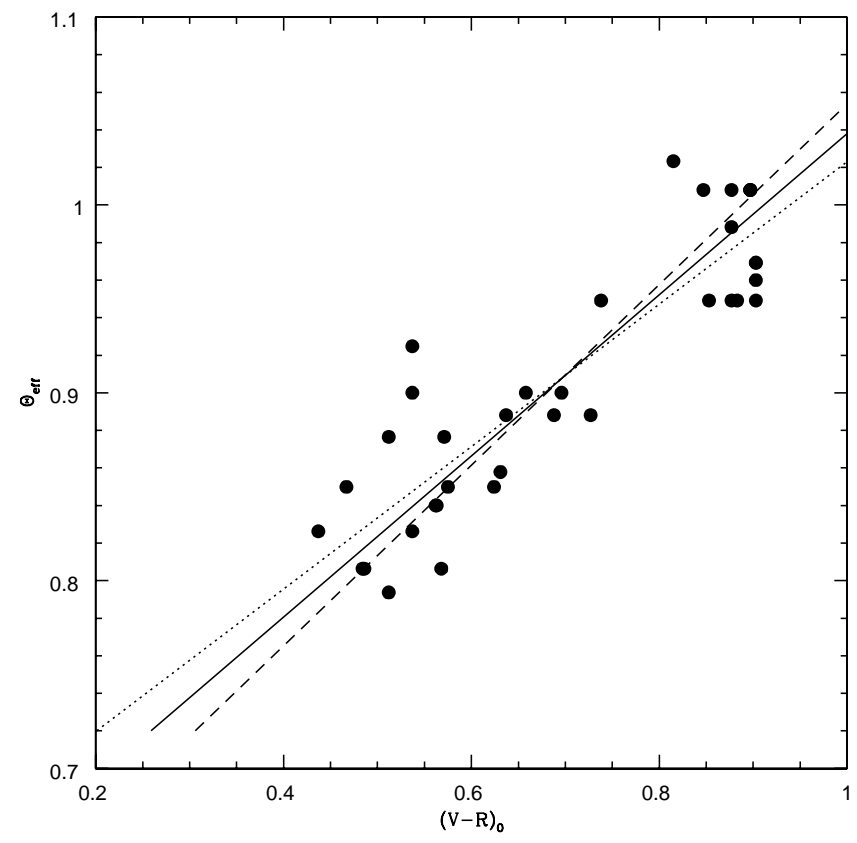

FIG. 3. $-\Theta_{\text {eff,spect }}$ vs. $(V-R)_{0}$

expect $\sigma=160 \mathrm{~K}$.) The plot of $\Delta T$ versus $T_{\text {spect }}$ is shown in Figure 4.

\subsection{Dependence on Gravity}

Synthetic colors (Kurucz 1993) also suggest that the slope of the color-temperature relation depends on gravity. In Figure 5, we plot the relation of synthetic $B-V$ colors and $\Theta_{\text {eff }}$ for gravities appropriate for Cepheids. The slope becomes flatter for lower gravities (longer period Cepheids). In our data, however, the change is harder to see. We can use Fernie's period-gravity relation (Fernie 1995) to estimate gravities for each of the Cepheids. Replotting Figure 2 to use different symbols to show Cepheids of different gravities (Fig. 6), we can investigate gravity sensitivity in the temperature scale. There is some weak evidence for a

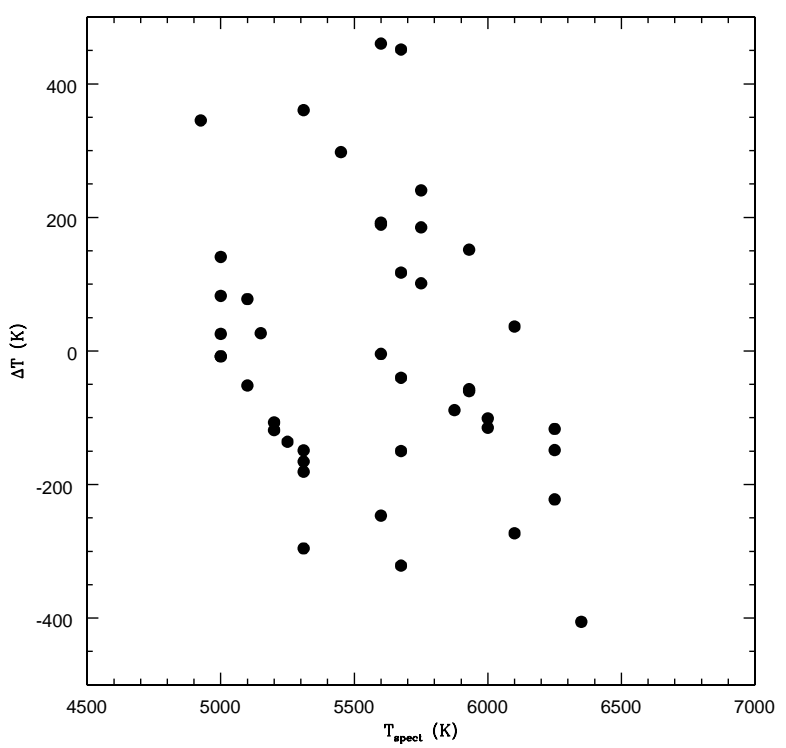

FIG. 4.-Differences between spectroscopic and photometric temperatures as a function of spectroscopic temperature. 
TABLE 2

Metallicity CoRrections to CePheid Colors

\begin{tabular}{|c|c|c|c|c|c|c|}
\hline$T_{\text {eff }}$ & $\log g$ & $\frac{d(B-V)}{d[\mathrm{Fe} / \mathrm{H}]}$ & $\frac{d(V-R)}{d[\mathrm{Fe} / \mathrm{H}]}$ & $\frac{d(V-I)}{d[\mathrm{Fe} / \mathrm{H}]}$ & $\frac{d(R-I)}{d[\mathrm{Fe} / \mathrm{H}]}$ & $\frac{d(V-K)}{d[\mathrm{Fe} / \mathrm{H}]}$ \\
\hline \multirow[t]{6}{*}{$4500 \ldots \ldots$} & 0.0 & +0.129 & -0.004 & -0.040 & -0.044 & -0.024 \\
\hline & 0.5 & +0.159 & +0.019 & -0.028 & -0.009 & +0.024 \\
\hline & 1.0 & +0.176 & +0.037 & -0.023 & +0.014 & +0.044 \\
\hline & 1.5 & +0.181 & +0.043 & -0.019 & +0.024 & +0.053 \\
\hline & 2.0 & +0.179 & +0.047 & -0.017 & +0.030 & +0.036 \\
\hline & 2.5 & +0.173 & +0.053 & -0.014 & +0.039 & +0.041 \\
\hline \multirow[t]{12}{*}{$4750 \ldots \ldots$} & 0.0 & +0.140 & +0.009 & -0.042 & -0.033 & -0.016 \\
\hline & 0.5 & +0.160 & +0.021 & -0.034 & -0.013 & +0.009 \\
\hline & 1.0 & +0.171 & +0.030 & -0.027 & +0.003 & +0.024 \\
\hline & 1.5 & +0.176 & +0.034 & -0.025 & +0.009 & +0.014 \\
\hline & 2.0 & +0.173 & +0.039 & -0.022 & +0.017 & +0.021 \\
\hline & 2.5 & +0.164 & +0.041 & -0.020 & +0.021 & +0.020 \\
\hline & 0.0 & +0.154 & +0.020 & -0.037 & -0.017 & -0.034 \\
\hline & 0.5 & +0.161 & +0.024 & -0.031 & -0.007 & -0.020 \\
\hline & 1.0 & +0.167 & +0.029 & -0.029 & -0.000 & -0.010 \\
\hline & 1.5 & +0.170 & +0.034 & -0.027 & +0.007 & -0.006 \\
\hline & 2.0 & +0.164 & +0.033 & -0.023 & +0.010 & +0.001 \\
\hline & 2.5 & +0.159 & +0.036 & -0.022 & +0.014 & -0.000 \\
\hline \multirow[t]{6}{*}{$5250 \ldots \ldots$} & 0.0 & +0.170 & +0.029 & -0.029 & -0.000 & -0.036 \\
\hline & 0.5 & +0.171 & +0.030 & -0.027 & +0.003 & -0.031 \\
\hline & 1.0 & +0.171 & +0.031 & -0.025 & +0.006 & -0.026 \\
\hline & 1.5 & +0.166 & +0.033 & -0.027 & +0.006 & -0.020 \\
\hline & 2.0 & +0.159 & +0.031 & -0.024 & +0.007 & -0.017 \\
\hline & 2.5 & +0.150 & +0.033 & -0.024 & +0.009 & -0.013 \\
\hline \multirow[t]{6}{*}{$5500 \ldots \ldots$} & 0.0 & +0.173 & +0.031 & -0.025 & +0.006 & -0.034 \\
\hline & 0.5 & +0.177 & +0.031 & -0.024 & +0.007 & -0.023 \\
\hline & 1.0 & +0.171 & +0.031 & -0.024 & +0.007 & -0.026 \\
\hline & 1.5 & +0.163 & +0.030 & -0.024 & +0.006 & -0.024 \\
\hline & 2.0 & +0.154 & +0.027 & -0.023 & +0.004 & -0.023 \\
\hline & 2.5 & +0.144 & +0.027 & -0.023 & +0.004 & -0.020 \\
\hline \multirow[t]{6}{*}{$5750 \ldots \ldots$} & 0.0 & +0.173 & +0.030 & -0.023 & +0.007 & -0.026 \\
\hline & 0.5 & +0.173 & +0.029 & -0.023 & +0.006 & -0.029 \\
\hline & 1.0 & +0.163 & +0.027 & -0.021 & +0.006 & -0.023 \\
\hline & 1.5 & +0.157 & +0.027 & -0.021 & +0.006 & -0.024 \\
\hline & 2.0 & +0.149 & +0.024 & -0.021 & +0.003 & -0.027 \\
\hline & 2.5 & +0.139 & +0.023 & -0.022 & +0.001 & -0.029 \\
\hline \multirow[t]{6}{*}{$6000 \ldots \ldots$} & 0.0 & +0.156 & +0.029 & -0.023 & +0.006 & -0.021 \\
\hline & 0.5 & +0.160 & +0.027 & -0.021 & +0.006 & -0.026 \\
\hline & 1.0 & +0.160 & +0.027 & -0.020 & +0.007 & -0.030 \\
\hline & 1.5 & +0.131 & +0.017 & -0.020 & -0.003 & -0.030 \\
\hline & 2.0 & +0.136 & +0.023 & -0.020 & +0.003 & -0.030 \\
\hline & 2.5 & +0.127 & +0.020 & -0.020 & -0.000 & -0.033 \\
\hline \multirow[t]{6}{*}{$6250 \ldots \ldots$} & 0.0 & +0.136 & +0.023 & -0.019 & +0.004 & -0.024 \\
\hline & 0.5 & +0.136 & +0.023 & -0.019 & +0.004 & -0.024 \\
\hline & 1.0 & +0.139 & +0.023 & -0.020 & +0.003 & -0.030 \\
\hline & 1.5 & +0.136 & +0.020 & -0.019 & +0.001 & -0.034 \\
\hline & 2.0 & +0.114 & +0.014 & -0.021 & -0.007 & -0.033 \\
\hline & 2.5 & +0.110 & +0.017 & -0.018 & -0.001 & -0.036 \\
\hline \multirow[t]{6}{*}{$6500 \ldots \ldots$} & 0.0 & +0.124 & +0.020 & -0.019 & +0.001 & -0.026 \\
\hline & 0.5 & +0.124 & +0.020 & -0.019 & +0.001 & -0.026 \\
\hline & 1.0 & +0.114 & +0.020 & -0.017 & +0.003 & -0.027 \\
\hline & 1.5 & +0.117 & +0.020 & -0.020 & -0.000 & -0.031 \\
\hline & 2.0 & +0.114 & +0.019 & -0.020 & -0.001 & -0.041 \\
\hline & 2.5 & +0.089 & +0.010 & -0.020 & -0.010 & -0.044 \\
\hline \multirow[t]{6}{*}{$6750 \ldots \ldots$} & 0.0 & +0.107 & +0.019 & -0.019 & -0.000 & -0.024 \\
\hline & 0.5 & +0.107 & +0.019 & -0.019 & -0.000 & -0.024 \\
\hline & 1.0 & +0.101 & +0.017 & -0.018 & -0.001 & -0.029 \\
\hline & 1.5 & +0.089 & +0.014 & -0.015 & -0.001 & -0.031 \\
\hline & 2.0 & +0.093 & +0.016 & -0.019 & -0.003 & -0.037 \\
\hline & 2.5 & +0.090 & +0.014 & -0.021 & -0.007 & -0.046 \\
\hline \multirow[t]{5}{*}{$7000 \ldots \ldots$} & 0.0 & +0.087 & +0.017 & -0.017 & -0.000 & -0.020 \\
\hline & 0.5 & +0.087 & +0.017 & -0.017 & -0.000 & -0.020 \\
\hline & 1.0 & +0.087 & +0.016 & -0.017 & -0.001 & -0.024 \\
\hline & 1.5 & +0.083 & +0.014 & -0.017 & -0.003 & -0.029 \\
\hline & 2.0 & +0.071 & +0.013 & -0.017 & -0.004 & -0.033 \\
\hline
\end{tabular}


TABLE 2-Continued

\begin{tabular}{ccccccc}
\hline \hline$T_{\text {eff }}$ & $\log g$ & $\frac{d(B-V)}{d[\mathrm{Fe} / \mathrm{H}]}$ & $\frac{d(V-R)}{d[\mathrm{Fe} / \mathrm{H}]}$ & $\frac{d(V-I)}{d[\mathrm{Fe} / \mathrm{H}]}$ & $\frac{d(R-I)}{d[\mathrm{Fe} / \mathrm{H}]}$ & $\frac{d(V-K)}{d[\mathrm{Fe} / \mathrm{H}]}$ \\
\hline $7250 \ldots \ldots$ & 0.0 & +0.070 & +0.014 & -0.015 & -0.001 & -0.017 \\
& 0.5 & +0.070 & +0.014 & -0.015 & -0.001 & -0.017 \\
& 1.0 & +0.069 & +0.013 & -0.016 & -0.003 & -0.020 \\
& 1.5 & +0.067 & +0.013 & -0.017 & -0.004 & -0.024 \\
& 2.0 & +0.061 & +0.010 & -0.016 & -0.006 & -0.030 \\
& 2.5 & +0.056 & +0.010 & -0.019 & -0.009 & -0.034 \\
\hline
\end{tabular}

gravity dependence in the slope of the color-temperature relation, but the gravity dependence is hard to model reliably. To look for the gravity dependence, we compare the color-temperature relations from the entire set of Cepheids (eqs. [7]-[8] above) with the color-temperature relations derived from only those Cepheids for which $1.0<\log g<2.0$. According to Fernie's period-gravity relation (Fernie 1995), these correspond to Cepheids for which

TABLE 3

Metallicity-CoRreCted, Dereddened Colors

\begin{tabular}{|c|c|c|c|c|c|c|c|}
\hline Cepheid & $\phi$ & $T_{\text {eff }}$ & $\log g$ & $(B-V)_{0}$ & $\Delta(B-V)^{\mathbf{a}}$ & $(V-R)_{0}$ & $\Delta(V-R)^{\mathrm{a}}$ \\
\hline \multirow[t]{2}{*}{ CF Cas ........ } & 0.655 & 5310 & 1.3 & 0.768 & -0.03 & 0.738 & -0.01 \\
\hline & 0.260 & 5600 & 1.7 & 0.687 & -0.03 & 0.658 & -0.01 \\
\hline \multirow[t]{2}{*}{ DL Cas....... } & 0.140 & 5875 & 1.5 & 0.689 & -0.02 & 0.631 & 0.00 \\
\hline & 0.389 & 5600 & 1.5 & 0.821 & -0.02 & 0.696 & 0.00 \\
\hline \multirow[t]{2}{*}{ V Cen.......... } & 0.236 & 5675 & 2.0 & 0.474 & -0.02 & $\ldots$ & 0.00 \\
\hline & 0.285 & 5600 & 2.0 & 0.514 & -0.02 & 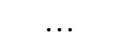 & 0.00 \\
\hline \multirow[t]{7}{*}{ T Mon......... } & 0.470 & 5310 & 1.1 & 1.285 & 0.02 & 0.877 & 0.00 \\
\hline & 0.387 & 5000 & 0.9 & 1.175 & 0.01 & 0.847 & 0.00 \\
\hline & 0.423 & 5000 & 0.9 & 1.225 & 0.01 & 0.877 & 0.00 \\
\hline & 0.461 & 5100 & 0.9 & 1.255 & 0.02 & 0.877 & 0.00 \\
\hline & 0.499 & 5000 & 0.9 & 1.275 & 0.01 & 0.897 & 0.00 \\
\hline & 0.536 & 5000 & 0.9 & 1.305 & 0.01 & 0.897 & 0.00 \\
\hline & 0.572 & 5000 & 0.9 & 1.305 & 0.01 & 0.897 & 0.00 \\
\hline \multirow[t]{2}{*}{ CV Mon....... } & 0.178 & 6250 & 1.5 & 0.534 & -0.01 & 0.568 & 0.00 \\
\hline & 0.363 & 5675 & 1.5 & 0.685 & -0.01 & 0.688 & 0.00 \\
\hline \multirow[t]{2}{*}{ S Nor .......... } & 0.550 & 5675 & 1.5 & 1.002 & 0.00 & $\ldots$ & 0.00 \\
\hline & 0.570 & 5600 & 1.5 & 1.002 & 0.00 & $\ldots$ & 0.00 \\
\hline TW Nor ....... & 0.056 & 5750 & 2.0 & 0.557 & 0.00 & $\ldots$ & 0.00 \\
\hline \multirow[t]{8}{*}{ U Sgr ......... } & 0.811 & 5675 & 2.0 & 0.793 & 0.00 & 0.637 & 0.00 \\
\hline & 0.958 & 6350 & 2.0 & 0.586 & 0.00 & 0.512 & 0.00 \\
\hline & 0.106 & 6100 & 2.0 & 0.468 & 0.00 & 0.437 & 0.00 \\
\hline & 0.254 & 5750 & 2.0 & 0.592 & 0.00 & 0.512 & 0.00 \\
\hline & 0.404 & 5600 & 2.0 & 0.685 & 0.00 & 0.537 & 0.00 \\
\hline & 0.152 & 5930 & 2.2 & 0.501 & 0.00 & 0.467 & 0.00 \\
\hline & 0.447 & 5450 & 1.7 & 0.715 & 0.00 & 0.537 & 0.00 \\
\hline & 0.363 & 6100 & 1.7 & 0.662 & 0.00 & 0.537 & 0.00 \\
\hline \multirow[t]{2}{*}{ WZ Sgr....... } & 0.950 & 5930 & 1.5 & 0.634 & -0.02 & 0.624 & 0.00 \\
\hline & 0.343 & 4925 & 0.7 & 1.068 & -0.02 & 0.815 & 0.00 \\
\hline EV Sct ......... & 0.695 & 5675 & 1.5 & 0.872 & -0.06 & 0.727 & -0.01 \\
\hline \multirow[t]{6}{*}{ SZ Tau ....... } & 0.017 & 6250 & 2.2 & 0.489 & 0.00 & 0.484 & 0.00 \\
\hline & 0.333 & 5930 & 2.2 & 0.632 & 0.00 & 0.575 & 0.00 \\
\hline & 0.647 & 6000 & 2.3 & 0.624 & 0.00 & 0.563 & 0.00 \\
\hline & 0.978 & 6250 & 2.2 & 0.470 & 0.00 & 0.486 & 0.00 \\
\hline & 0.291 & 6000 & 2.0 & 0.615 & 0.00 & 0.562 & 0.00 \\
\hline & 0.603 & 5750 & 2.0 & 0.646 & 0.00 & 0.571 & 0.00 \\
\hline \multirow{2}{*}{ SW Vel ........ } & 0.348 & 5150 & 1.0 & 1.145 & -0.01 & $\ldots$ & 0.00 \\
\hline & 0.616 & 5100 & 0.7 & 1.144 & -0.01 & $\ldots$ & 0.00 \\
\hline \multirow[t]{6}{*}{ SV Vul........ } & 0.260 & 5310 & 0.7 & 1.158 & 0.01 & 0.853 & 0.00 \\
\hline & 0.283 & 5310 & 0.6 & 1.172 & 0.01 & 0.883 & 0.00 \\
\hline & 0.305 & 5310 & 0.9 & 1.185 & 0.01 & 0.903 & 0.00 \\
\hline & 0.327 & 5250 & 0.9 & 1.198 & 0.01 & 0.903 & 0.00 \\
\hline & 0.350 & 5200 & 0.7 & 1.216 & 0.01 & 0.903 & 0.00 \\
\hline & 0.371 & 5200 & 0.8 & 1.226 & 0.01 & 0.903 & 0.00 \\
\hline
\end{tabular}

${ }^{\text {a }} \Delta(B-V)$ and $\Delta(V-R)$ are changes to dereddened colors for nonsolar metallicities. 


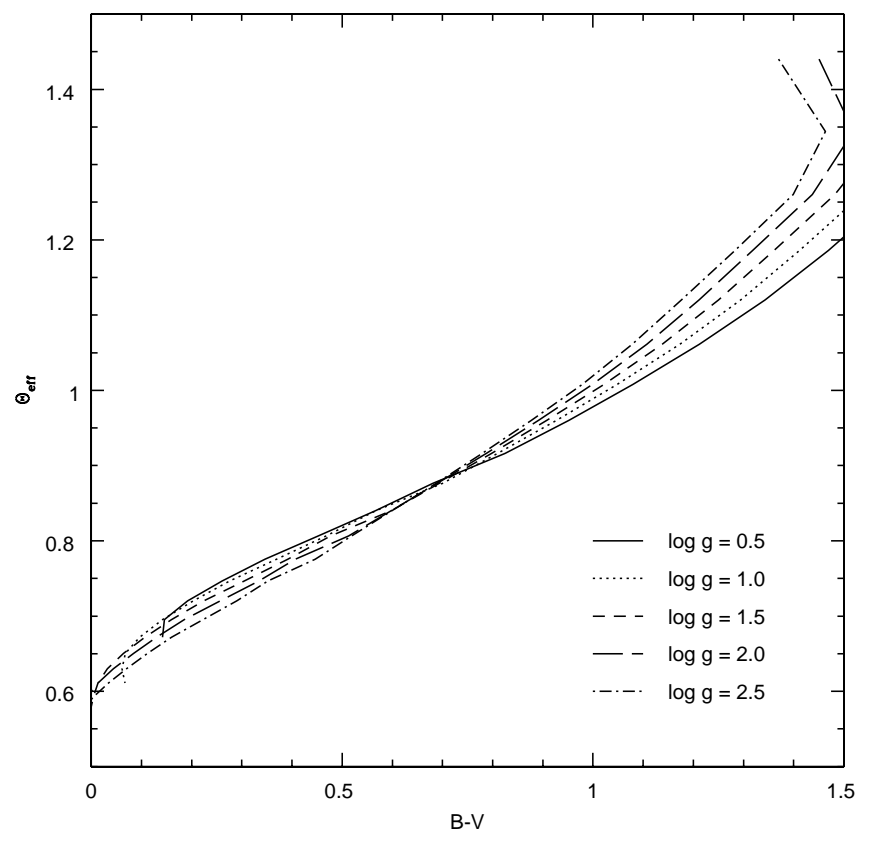

FIG. 5.-Synthetic color-temperature relations for different gravities.

3.5 days $<P<26$ days. We find

$$
\begin{gathered}
\Theta_{\text {eff }}=(0.714 \pm 0.026)+(0.231 \pm 0.034)(B-V)_{0}, \\
\Theta_{\text {eff }}=(0.600 \pm 0.049)+(0.448 \pm 0.079)(V-R)_{0} .
\end{gathered}
$$

Color-temperature relations for $1.0<\log g<2.0$ have the same slope as color-temperature relations for the entire set of Cepheids, demonstrating that the gravity dependence is small.

\section{OTHER CEPHEID TEMPERATURE STUDIES}

Many authors have formed supergiant $B-V$ versus $T_{\text {eff }}$ relations from studies of Cepheids and nonvariable super-

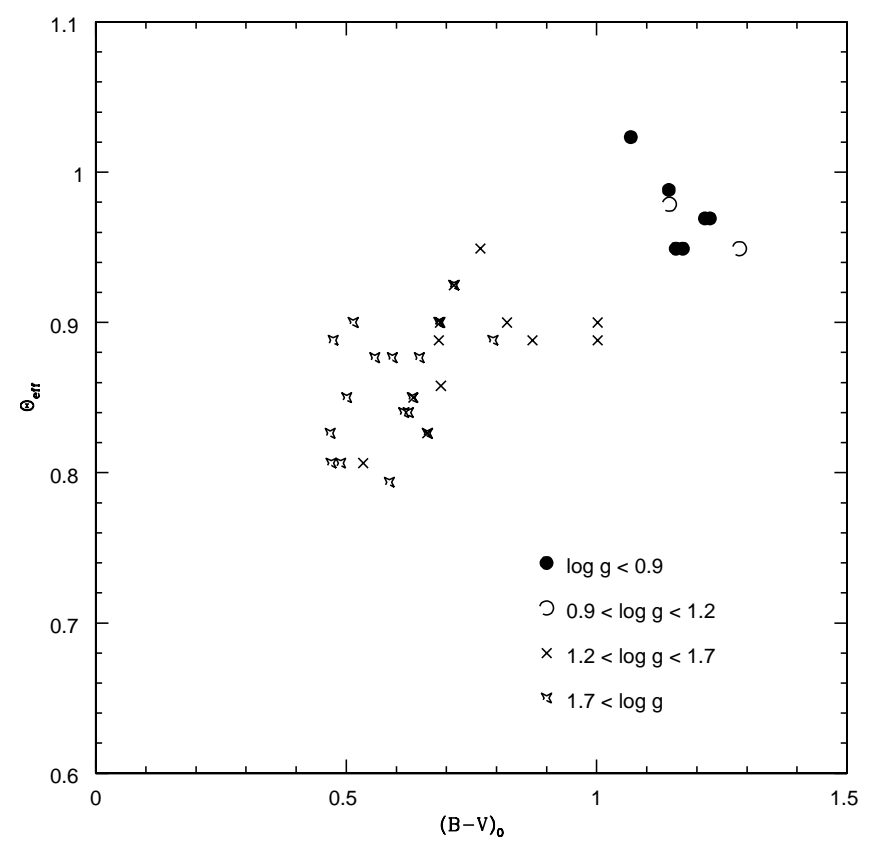

FIG. 6.-Our color-temperature relation with gravity dependence indicated. Symbols have been plotted for stars for which we have at least three spectroscopic gravity determinations.

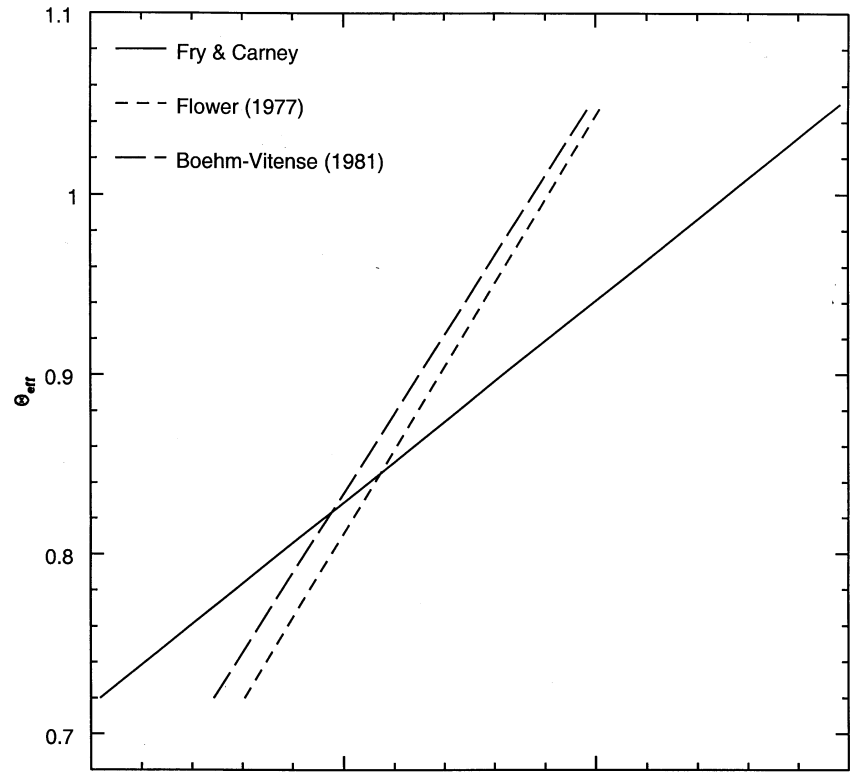

FIG. 7.-Previous Cepheid color-temperature relations are shown with ours.

giants. We show the results of previous studies and our results in Figure 7. Other summaries are in Pel (1985) and Teays (1986). To compare with previous work, we also express equation (7) in terms of $\log T_{\text {eff }}$ (Fig. 8):

$$
\log T_{\text {eff }}=(3.837 \pm 0.007)-(0.108 \pm 0.008)(B-V)_{0} .
$$

For the following discussion, we restrict comparisons to the temperature scales that included cluster Cepheids in their calibrations.

Schmidt (1972) also used spectrum scanning to obtain energy distributions for 20 Cepheids, including one cluster Cepheid (SZ Tau) for which we have spectroscopic tem-

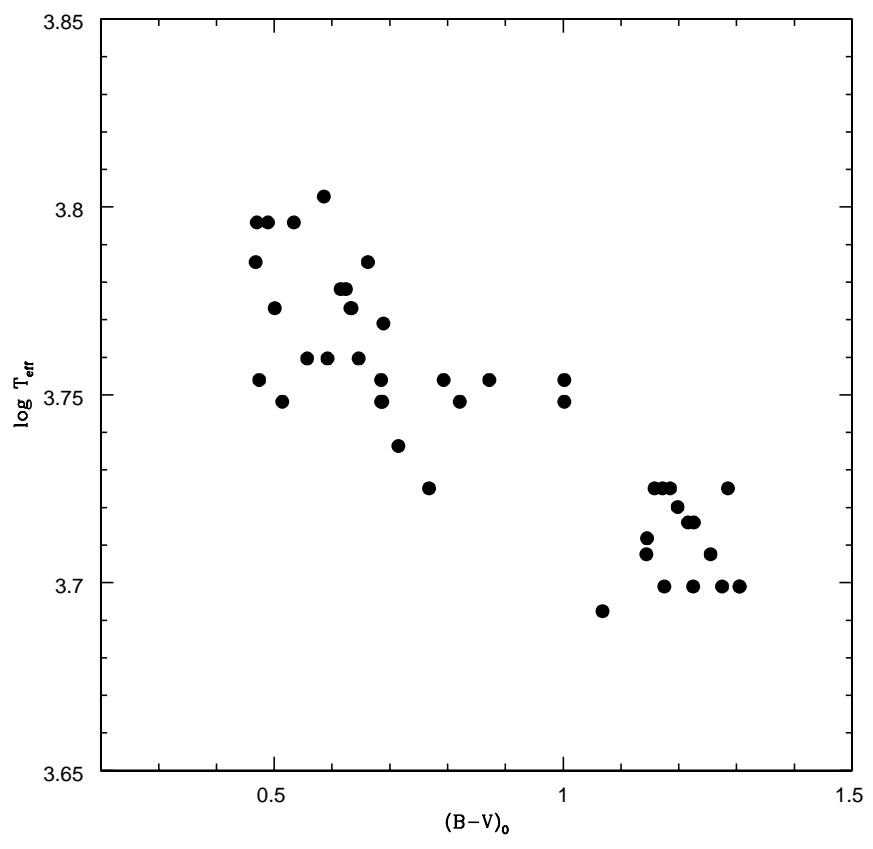

FIG. 8. - Plot of $\log T_{\text {eff }}$ vs. $(B-V)_{0}$. 


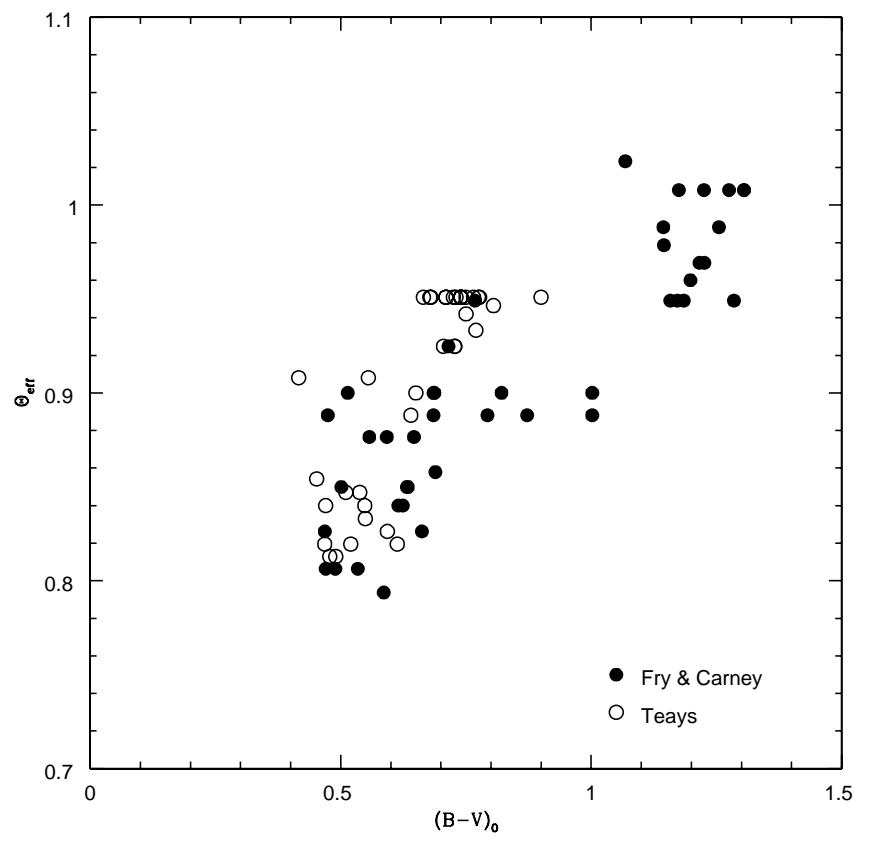

FIG. 9. $-T_{\text {eff }}$ vs. $(B-V)_{0}$ : comparison with Teays's results.

peratures at the same phase. The difference between our temperature and Schmidt's is $-74 \mathrm{~K}$.

Flower (1977) used a variety of published temperatures to derive a supergiant color-temperature relation. Using the data in his Table 1 , we find

$$
\Theta_{\text {eff }}=(0.579 \pm 0.007)+(0.464 \pm 0.008)(B-V)
$$

for $0.3<B-V<1.3$, the range of Cepheid colors. BöhmVitense (1981), in a review of stellar temperatures, gives a supergiant color-temperature relation based on a variety of direct and indirect temperature determinations. Using her Table 4, we find

$$
\Theta_{\text {eff }}=(0.613 \pm 0.003)+(0.441 \pm 0.004)(B-V)
$$

for $0.3<B-V<1.3$. This is very different from our relation. However, Böhm-Vitense (1981) notes that, for the same $B-V$ color, different authors found temperatures that disagree by as much as $400 \mathrm{~K}$. Hence the errors in equation (13) are probably underestimates. It is also worth noting that Flower's and Böhm-Vitense's temperature scales are not independent, because they both rely on the earlier work of van Paradijs (1973) to determine the low end of their temperature scales. Van Paradijs (1973) derived abundances and atmospheric parameters of seven nonvariable $\mathrm{G}$ and $\mathrm{K}$ supergiants using an extrapolation of the model atmospheres of Carbon \& Gingerich (1969). It is not surprising that the newer Kurucz models we employed are different from older model atmospheres for cool stars, for which line blanketing is important (see below).

Teays (1986) obtained energy distributions for six Cepheids, CF Cas, DL Cas, CV Mon, S Nor, EV Sct, and U Sgr, at several phases to form a Cepheid $B-V$ versus $T_{\text {eff }}$ relation. He obtained spectrophotometry of the Cepheids and compared with model atmospheres to derive temperatures. Teays used the older Kurucz grid of lineblanketed model atmospheres (Kurucz 1979), which did not contain models cooler than $T_{\text {eff }}=5500 \mathrm{~K}$. For $5300 \mathrm{~K}<$ $T_{\text {eff }}<5500 \mathrm{~K}$, Teays extrapolated the coolest models. $\mathrm{He}$ assumed solar metallicity for all of the Cepheids. Corrections for nonsolar metallicity will make a small difference for EV Sct $([\mathrm{Fe} / \mathrm{H}]=-0.37)$ and $\mathrm{CF}$ Cas $([\mathrm{Fe} / \mathrm{H}]=-0.20)$. His results are shown with ours in Figure 9. At the high-temperature end, the agreement between the two temperature scales is quite good. At the low-temperature end, the two scales do not agree. Disagreement at the low-temperature end, especially in the temperature range where Teays extrapolated the older Kurucz models, is not surprising. The older Kurucz models were atomic-line blanketed $\left(\sim 10^{6}\right.$ lines) but did not contain contributions from molecular lines, which are important for cooler stars. The new models include both atomic and molecular line blanketing $\left(\sim 50 \times 10^{6}\right.$ lines $)$.

\section{SUMMARY}

We derived Cepheid color-temperature relations using our spectroscopic temperatures (Fry \& Carney 1997) and published photometry. We have corrected the observed colors both for reddening and to solar metallicity. The effect of gravity on the colors is small. We find that $B-V$ and $V-R$ are the best colors to use to determine a Cepheid photometric temperature scale. The color-temperature relations we found are given by equations (7), (8), and (11). The color-temperature relations will be useful for future Cepheid Baade-Wesselink studies and for abundance analyses of more Galactic and extragalactic Cepheids.

We thank an anonymous referee for alerting us to the importance of metallicity corrections to the derivation of the reddening estimates and a second anonymous referee for pointing out that $E(\mathrm{OB}) \neq E($ Cepheid). This work was done as part of A. M. F.'s Ph.D. thesis at the University of North Carolina. A. M. F. would like to thank the Carolina Society of Fellows for Royster Dissertation Fellowship support. We also gratefully acknowledge financial support from the National Science Foundation grant AST 92-21237 to the University of North Carolina.

\section{REFERENCES}

Böhm-Vitense, E. 1981, ARA\&A, 19, 295

Carbon, D., \& Gingerich, O. 1969, in Stellar Atmospheres, ed. O. Gingerich (Cambridge: MIT Press), 377

Carney, B. W., Laird, J. B., Latham, D. W., \& Kurucz, R. L. 1987, AJ, 94, 1066

Feast, M. W., \& Walker, A. R. 1987, ARA\&A, 25, 1987

Fernie, J. D. 1995, AJ, 110, 2361

Flower, P. J. 1977, A\&A, 54, 31

Fry, A. M., \& Carney, B. W. 1997, AJ, 113, 1073

Isobe, T., Feigelson, E. D., Akritas, M. G., \& Babu, G. J. 1990, ApJ, 364, 104

Jones, R. V. 1988, ApJ, 326, 305
Kurucz, R. L. 1979, ApJS, 40, 1

1993, ATLAS9 Stellar Atmosphere Programs and $2 \mathrm{~km} / \mathrm{s}$ Grid (Cambridge: Smithsonian Astrophys. Obs.)

Laird, J. B., Carney, B. C., \& Latham, D. W. 1988, AJ, 95, 1843

Laney, C. D., \& Stobie, R. S. 1992, A\&AS, 93, 93

Moffett, T. J., \& Barnes, T. G. 1980, ApJS, 44, 427 1984, ApJS, 55, 389

Oke, J. B. 1961a, ApJ, 133, 90

1961b, ApJ, 134, 214

Pel, J. W. 1985, in Cepheids: Theory and Observations, ed. B. F. Madore (Cambridge: Cambridge Univ. Press), 1

Schmidt, E. G. 1972, ApJ, 174, 605 
Storm, J., Carney, B. W., \& Latham, D. W. 1994, A\&A, 290, 443

Teays, T. J. 1986, Ph.D. thesis, Univ. Nebraska

Turner, D. G. 1992, AJ, 104, 1865

Turner, D. G., Forbes, D., \& Pedreros, M. 1992, AJ, 104, 1132

Turner, D. G., van den Bergh, S., Younger, P. F., Danks, T. A., \& Forbes, D. 1993, ApJS, 85, 119 van Paradijs, J. 1973, A\&A, 23, 369

Welch, D. L., Wieland, F., McAlary, C. W., McGonegal, R., Madore, B. F., McLaren, R. A., \& Neugebauer, G. 1984, ApJS, 54, 547 